\title{
Discussion on Improving Quality of Service through Available Bandwidth Estimation in Mobile Ad Hoc Networks
}

\author{
K. Mohideen Vahitha Banu \\ Department of Computer Science, P.S.G.R. Krishnammal College for Women \\ Bharathiar University \\ Coimbatore, India
}

\begin{abstract}
The term bandwidth refers to the data rate that a network link or a network path can transfer. Bandwidth is one of the guarantee attribute to measure the performance of the network. Estimating accurate available bandwidth allows a node to make optimal decision before sending a packet in networks. It is therefore obvious that the available bandwidth estimation improves the Quality of Service (QoS) in wired and wireless Networks. Measuring available bandwidth in ad hoc networks is challenging issue in Mobile Ad hoc Network (MANET). This paper describes various tools and techniques to measure the available bandwidth and purpose of bandwidth estimation to improve the QoS in MANET.
\end{abstract}

Keywords: Available Bandwidth, MANET, QoS

\section{INTRODUCTION}

A Mobile Ad hoc Network (MANET) is an autonomous and decentralized system of mobile nodes which are connected by wireless links. They do not need any infrastructure. This kind of networks are very flexible and suitable for several situations and applications, thus they allow the establishing of temporary communication without pre-installed infrastructure. IEEE has defined for wireless technology called IEEE 802.11 standard. This standard provides an ad hoc mode, allowing mobiles to communicate directly. No standardized mechanism has been developed to measure the remaining resources in ad hoc networks [1]. Utilizing resources in systematic way without wastage will increase the network throughput. Bandwidth is one of the main resources in network traffic. So it is ardent fact that estimating accurate available bandwidth improves the network performance and Quality of Service in networks automatically.

The term Quality of Service (QoS) refers to resource reservation control mechanisms rather than the achieved service quality. Quality of service is the ability to provide different priority to different applications, users, or data flows, or to guarantee a certain level of performance to a data flow [2]. For example in video conferencing which needs to send millions of bits per second to refresh a color screen while the total number of bits in an e-mail may not reach even a million. The efficient QoS should provide support for these types of applications. Although resource estimation is challenging task in ad hoc networks, it should be taken into account to improve the QoS of networks. The network is expected to guarantee a set of measurable pre-specified service attributes to the users in terms of end-to- end performance, such as delay, bandwidth, probability of packet loss, delay variance (jitter), etc. Estimating accurate bandwidth increases overall network performance.

Available bandwidth is estimated in ad hoc networks based on the IEEE 802.11 MAC Layer. In ad hoc network, a host's available bandwidth refers to amount of bandwidth available to the node to transmit packets to the network. Whole channel will not be used for packet transmission. The amount of bandwidth needs to further communication such as initiating communication, neighbor's interferences etc which reduces the node's available network. So every node should know the status of network for taking optimum decision. Therefore bandwidth estimation is an important action that is needed to provide QoS in MANETs. However, bandwidth estimation is extremely difficult; because each host has imprecise knowledge of the network status and links change dynamically in ad hoc networks. Therefore, an effective bandwidth estimation scheme is highly desirable.

The rest of the paper is organized as follows. Section II presents the detailed about the bandwidth estimation. The detailed survey on the available bandwidth estimation is presented in section III. Section IV describes the considerations when estimating available bandwidth. Finally, proposed work is presented in conclusion.

\section{BANDWIDTH ESTIMATION}

The 802.11 protocol covers the MAC and Physical Layer. The basic access mechanism, called Distributed Coordination Function (DCF). In wireless networks the radio channel of each node is shared with all its neighbors. Because of the shared medium, a node can successfully use the channel only when all its neighbors do not transmit and receive packets at the same time. Due to the shared nature of wireless network communication and MAClayer mechanisms, a node can estimate the channel occupancy by monitoring its environment. The radio medium is pervasive and a frame emission from a node has an impact on other nodes that located in common communication area.

The term bandwidth refers to the information carrying capacity of a node in networks. It is measured by bits per second which refers to the speed of bit transmission in a channel or link. The bandwidth related metrics [1] are Capacity, Available Bandwidth and Bulk Transfer capacity (BTC). The first two are defined both for individual links and end-to-end paths, while the BTC is usually defined only for an end-to-end path. The maximum possible bandwidth that a link or path can deliver is called Capacity. The maximum unused bandwidth at a link or path is called available bandwidth. The achievable throughput of a bulktransfer TCP connection is called BTC. Here Available bandwidth is focused as QoS metric. 
Available bandwidth is the amount of bandwidth left over after the cross traffic. It can be determined by finding the time period for which the link is not utilized for transmitting data. Estimation of available bandwidth is difficult task in ad hoc networks. The general formula for estimating Available Bandwidth is given in 1 .

BWavailable $=$ BWphysical - BWconsumed

When the available bandwidth is estimated, the activities of the neighbors of nodes must be taken into account, since the wireless medium of a node is shared among neighboring nodes. Various tools and techniques are developed and proposed in [10] for estimating available bandwidth in networks. These are categorized into active and passive techniques. These are discussed in next section.

\section{THE SURVEY ON AVAILABLE BANDWIDTH ESTIMATION}

Available bandwidth is estimated in both wired and wireless networks. Available bandwidth estimation techniques are classified into two categories. These are intrusive and passive bandwidth estimation techniques. Intrusive techniques mostly used in wired networks. Passive techniques are used in ad-hoc networks.

\subsection{Active Bandwidth Estimation Techniques}

The techniques that are based on the end-to-end probe packets to estimate available bandwidth in wired networks. Numbers of techniques were developed to measure the end-to-end available bandwidth by sending packets of equal size from a source to receiver. Many available bandwidth estimation tools [3] have emerged such as Pathload, TOPP, PTR/IGI, Delphi, and Pathchirp. These techniques that relay on the emission of dedicated end-to-end probe packets to estimate the available bandwidth along a path.

Cprobe was the first tool to attempt to measure endto-end available bandwidth. Cprobe measures the dispersion of a train of eight maximum-sized packets. In general the dispersion rate depends on all links in the path as well as on the train's initial rate. In contrast the available bandwidth only depends on the tight link of the path. In [4], Cprobe is described as a pioneering tool for estimating the available bandwidth using end-to-end measurements. Cprobe doesn't assume fair queuing. Instead of using a pair of packets, Cprobe sends a short train of ICMP packets and computes the available bandwidth as the probe traffic divided by the interval between the arrival of the last ICMP ECHO and the first ICMP ECHO in the train.

In [3], the active available bandwidth measurement tool is presented which is called Spruce (Spread PaiR Unused Capacity Estimate). In which presented Spruce is a tool for end hosts to measure available bandwidth. It samples the arrival rate at the bottleneck by sending pairs of packets spaced so that the second probe packet arrives at a bottle- neck queue before the first packet departs the queue. Spruce then calculates the number of bytes that arrived at the queue between the two probes from the inter-probe spacing at the receiver. Spruce computes the available bandwidth as the difference between the path capacity and the arrival rate at the bottleneck. Spruce was assumed that which is fairly accurate in realistic Internet settings than IGI and PATHLOAD tools.
In [5] the active probing tool PathChirp is described for estimating available bandwidth in wired network. PathChirp obtains a rich set of information from which to dynamically estimate the available bandwidth. Since it uses only packet interarrival times for estimation, pathChirp does not require synchronous nor highly stable clocks at the sender and receiver. sender and receiver.

The above mentioned active techniques do not yield accurate results in a wireless ad hoc context. They do not consider the need for preserving existing flow service level when computing the available bandwidth. They also introduce additional traffic in the network that may disturb the network operation and simultaneous measurements may interfere.

\subsection{Passive Bandwidth Estimation Techniques}

The techniques which are using only local information for the utilization of the bandwidth. A typical example of such approaches is a node monitoring the channel usage by sensing the radio medium. These mechanisms are usually transparent, but they may exchange information via one-hop broadcasts, as such information can be piggybacked in the Hello messages used by many routing protocols to discover the local topology. In [6], bandwidth reservation protocol is proposed which is called Bandwidth Reservation under InTerferences (BRuIT) that takes into account the notion of carrier sensing area in the available bandwidth estimation. Indeed, with CSMA protocols (like in IEEE 802.11), two nodes within carrier sensing range share the medium and thus the bandwidth, even if they cannot directly communicate. Therefore, each node needs not only to know the channel occupancy in its communication range, but also in its carrier sensing range. BRuIT attempts to compute the channel usage in the carrier sensing area. In BRuIT, the carrier sensing area is approximated by the two-hop neighborhood. The drawback of this method is that the two-hop neighborhood may not correspond exactly to the carrier sensing area.

In [7], the Contention Aware Admission Control Protocol (CACP) is proposed. The goal is, like in BRuIT, to determine the available bandwidth of the nodes in the carrier sensing area. First, each node computes the local idle channel time fraction by a permanent monitoring of the radio medium. Then, the authors propose three different techniques: to use, like in BRuIT, Hello messages to broadcast this information over the two-hop neighborhood; to increase the transmission power of nodes such that all the nodes in the carrier sensing area could be reached; or to reduce the sensitivity of the mobiles in order that each node takes into account the bandwidth used in its carrier sensing area.

In the protocol AAC [8], each node estimates its local used bandwidth by simply adding the size of sent and sensed packets over a fixed period of time. The packet size is computed by estimating the medium occupancy time. Therefore this method considers data sent in the carrier sensing area. Finally, the link available bandwidth is the minimum available bandwidth of all nodes belonging to the carrier sensing areas of the sender and the receiver. AAC also estimates the contention count of nodes along a QoS path to solve intra contention problem.

Finally, ABE technique to estimate the available bandwidth in IEEE 802.11 based ad-hoc networks in [9]. This method uses the carrier sense capability combined to other techniques such as collision prediction to perform this estimation. The few constraints are considered when estimating the available bandwidth from a node to one of its neighbors. These are carrier sense mechanism, Link's available bandwidth estimation, 
collision detection and backoff detection. The carrier sense mechanism prevents two close emitters from transmitting simultaneously, unless they draw the same backoff counter value. Therefore, an emitter shares the channel bandwidth with all its close neighbors. The channel utilization has to be monitored to evaluate the capacity of a node to emit a given traffic volume.

For a transmission to take place, the receiver needs that no interference occurs during the whole transmission. Therefore, the value of the available bandwidth on a link depends on both peer channel utilization ratios and also on the idle period synchronization. This synchronization needs to be evaluated. No collision detection is possible in a wireless environment. Therefore, whenever a collision happens, both colliding frames are completely emitted, maximizing the bandwidth loss. Finally, when collisions happen on unicast frames, the IEEE 802.11 protocol automatically retries to emit the same frame, drawing the backoff counter in a double-sized contention window. The time lost in the additional overhead may also have an impact on the available bandwidth and has to be evaluated.

\section{ACCURATE AVAILABLE BANDWIDTH ESTIMATION}

From the above literature study, some of characteristics that were identified for bandwidth estimation from one node to its neighbors and detailed evaluation are presented in [9]. These are following.

\subsection{Carrier sense mechanism}

The node is first needed to contend for medium access whenever it sends frame in network. If channel is free, it can send a frame. Therefore, a sender needs to evaluate the proportion of time the medium is idle to determine the chance to gain access to the shared resource. So the carrier sense mechanism prevents two close emitters from transmitting simultaneously, unless they draw the same backoff counter value. Therefore, an emitter shares the channel bandwidth with all its close neighbors. The expected delay should be computed for accurate bandwidth estimation.

\subsection{Channel's idle period synchronization}

For a transmission to take place, the receiver needs that no interference occurs during the whole transmission. Therefore, the value of the available bandwidth on a link depends on both peer channel utilization ratios and also on the idle period synchronization. This synchronization needs to be evaluated.

\subsection{Collision Probability}

No collision detection is possible in a wireless environment. Therefore, whenever a collision happens, both colliding frames are completely emitted maximizing the bandwidth loss. The collision probability is needed to be estimated and integrated to Available bandwidth estimation.

\subsection{Backoff Time}

When collisions happen on unicast frames, the IEEE 802.11 protocol automatically retries to emit the same frame, drawing the backoff counter in a double-sized contention window. The time lost in the additional overhead may also have an impact on the available bandwidth and has to be evaluated. Commonly resolving the collisions is handled by binary exponential backoff scheme in MAC Layer. Ignoring backoff account provides high inaccuracy in the estimated available bandwidth.

\section{CONCLUSION}

From the above detailed study and survey, Available bandwidth is an essential parameter for measuring network performance is clearly understood. Estimating available bandwidth allows a node to make efficient decision before sending a frame in networks. From the above presented work, backoff time takes much contribution in avoiding collision in wireless networks. This process is handled by binary exponential algorithm (BEB). In this algorithm the contention window is doubled when the channel is busy. Reaching such large window sizes decreases the expected wait time for a given node to access to the shared medium. Moreover, a large window size tends to contribute to increasing channel idle times, leading to a major waste in the shared channel bandwidth

Above study motivated to propose the Fibonacci backoff algorithm instead of BEB algorithm in $\mathrm{ABE}$ technique to estimate the accurate bandwidth to improve the overall performance of the network. In Fibonacci backoff algorithm, the contention window increases gradually when the channel is busy. Therefore, more channel idle time is reduced and it allows neighbor's node access the channel frequently.

\section{REFERENCES}

[1] Cheikhsarr, ClaudeChaudet, Guillaume Chelius, IsabellGuérin Lassous,'Improving Accuracy in Available BandwidthEstimatiofor 802.11-based Ad Hoc Networks", 2006.

[2] Prof. Dr. Gustavo Alonso, "Quality of Service for Mobile AdHoc Networks"

[3] Jacob Strauss, Dina Katabi, Frans Kaashoek, "A Measurement Study of Available Banwidth Estimation Tools

[4] R. L. Carter and M. E. Crovella. Dynamic Server Selection Using Bandwidth Probing in Wide-Area Networks. Technical Report TR-96-007, Boston University Computer Science Department, 1996.

[5] Vinay J. Ribeiro, Rudolf H. Riedi, "pathChirp: Efficient Available Bandwidth Estimation for Network Paths"

[6] Chaudet and I.G. Lassous, "BRuIT-Bandwidth Reservation under InTerferences Influence," Proc. Europeon Wireless (EW '02),Feb. 2002

[7] Yang and R. Kravets, "Contention Aware Admission control for Adhoc Networks", IEEE Trans. Mobile Computing, vol.4, pp 363-377,2005

[8] R. de Renesse, M. Ghassemian, V. Friderikos, and A.H. Aghvami, "Adaptive Admission Control for Ad Hoc and Sensor Networks Providing Quality of Service," technical report, King College London, May 2005.

[9] Cheikh Sarr, Claude Chaudet, Guillaume Chelius, and Isabelle Gue'rin Lassous," Bandwidth Estimation for IEEE 802.11Based Ad Hoc Networks", IEEE Trans. Mobile Computing, vol. 7,No. 10, October 2008.

[10] R. S. Prasad, M.Murray, C. Dovrolis, K. Claffy_B "Bandwidth estimation: metrics, measurement techniques, and Tools", IEEE Network, vol. 17,no. 6, pp. 27-35, Nov. 2003. 